\title{
BMJ Open Experiences of digital communication with automated patient interviews and asynchronous chat in Swedish primary care: a qualitative study
}

\author{
Artin Entezarjou (D) , Beata Borgström Bolmsjö (D) , Susanna Calling (D) , \\ Patrik Midlöv (D) , Veronica Milos Nymberg (i)
}

To cite: Entezarjou A, Bolmsjö BB, Calling S, et al. Experiences of digital communication with automated patient interviews and asynchronous chat in Swedish primary care: a qualitative study. BMJ Open 2020;10:e036585. doi:10.1136/ bmjopen-2019-036585

- Prepublication history and additional material for this paper are available online. To view these files, please visit the journal online (http://dx.doi. org/10.1136/bmjopen-2019036585).

Received 24 December 2019 Revised 27 April 2020 Accepted 17 June 2020
D) Check for updates

(c) Author(s) (or their employer(s)) 2020. Re-use permitted under CC BY-NC. No commercial re-use. See rights and permissions. Published by BMJ.

Department of Clinical Sciences Malmö, Lund University, Lund, Sweden

Correspondence to Dr Artin Entezarjou; artin.entezarjou@med.lu.se

\section{ABSTRACT}

Objectives To explore staff experiences of working with a digital communication platform implemented throughout several primary healthcare centres in Sweden.

Design A descriptive qualitative approach using focus group interviews. Qualitative content analysis was used to code, categorise and thematise data.

Setting Primary healthcare centres across Sweden, in both rural and urban settings.

Participants A total of three mixed focus groups, comprising 19 general practitioners and nurses with experience using a specific digital communication platform.

Results Five categories emerged: 'Fears and Benefits of Digital Communication', 'Altered Practice Workflow', 'Accepting the Digital Society', 'Safe and Secure for Patients' and 'Doesn't Suit Everyone and Everything'. These were abstracted into two comprehensive themes: 'Adjusting to a novel medium of communication' and 'Digitally filtered primary care', describing how staff experienced integrating the software as a useful tool for certain clinical contexts while managing the communication challenges associated with written communication.

Conclusions Family medicine staff were ambivalent concerning the use of digital communication but, after a period of adjustment, it was seen as a useful communication tool especially when combined with continuity of care. Staff acknowledged limitations regarding use by inappropriate patient populations, information overload and misinterpretation of text by both staff and patients.

\section{INTRODUCTION}

The patient interview and physical examination are central to family medicine consultations. In Sweden, patients are increasingly using digital communication to access primary care. ${ }^{1}$ Swedish healthcare holds a high international standard, ${ }^{2}$ but low continuity and poor accessibility to primary care contribute to low patient satisfaction. ${ }^{3}$ Whether digital communication can address or aggravate these challenges is currently
Strengths and limitations of this study

- This is the first focus group study describing both physicians' and nurses' experiences of two-way digital communication between patients and providers in primary care settings.

- Theoretical saturation and high participant engagement allowed for rich descriptions and transferability of our findings to other contexts.

- Limitations include lack of multiple coders and a potential bias toward physician perspectives as the interviewers were both physicians.

unknown. ${ }^{4}$ Furthermore, staffs' low technical literacy and resistance to change may be common barriers to implementation, ${ }^{5}$ limiting potential benefits of such technology from being realised.

Heterogeneity between digital communication tools is high, making it difficult to draw general conclusions about their usefulness. Some use synchronous video communication, while others are asynchronous 'chat-based'. Different variations of automated patient interviewing software can also be used to gather key information prior to consultations.

The current study evaluates a digital communication platform (developed by Doctrin $A B$, referred to as 'the platform' in this paper) implemented across several primary healthcare centres (PHCCs) in Sweden for use as an alternative point of access to primary care. Patients choose among a prespecified list of queries and access an automated patient interviewing software on their computer, tablet or smartphone, freely writing their ideas, concerns and expectations as is common in family medicine consultations. ${ }^{6}$ They then answer a query-specific questionnaire, including the possibility to attach images, with answers presented to the healthcare provider (usually a nurse) who can 
proceed to communicate via asynchronous chat-based two-way communication. General practitioners (GPs) or other staff can join the chat if required. If a query cannot be concluded via digital communication, the patient is scheduled for a relevant physical appointment.

A Norwegian study recently found that GPs generally had positive experiences with using digital communication. ${ }^{7}$ Meanwhile, UK studies found that GPs felt such communication benefitted the patients and saved time, but GPs also raised concerns about security, increased workloads and poor integration into clinical practice. ${ }^{89}$

None of the above studies evaluated two-way digital communication systems, where both the patient and the provider can send digital messages. Such communication has been studied in the context of specific diseases ${ }^{10-12}$ or mobile phone text messaging without an adapted platform software. ${ }^{13}$

Furthermore, leveraging reports summarising patient ideas, concerns and expectations prior to digital communication may be important for staff to more effectively help patients without additional workloads. ${ }^{8}$ Therefore this qualitative study aimed to answer the following research question:

How do family medicine physicians and nurses experience the implementation and use of digital communication in the form of automated patient interviewing software and chat-based patient-provider communication?

\section{METHODS}

\section{Qualitative approach and research paradigm}

This study deemed an interpretivist paradigm suitable for understanding the phenomena of staff experience working with digital communication. ${ }^{14}$ Focus group interviews, commonly used to study attitudes and needs of medical staff, ${ }^{15}$ were thus chosen as the data-collection method. GPs and nurses form pre-existing groups working together as a team during focus group interviews, allowing for 'naturalistic' exchanges during data collection. This may give a deeper understanding of the target phenomenon. Open discussions allow participants to debate the studied phenomenon from a personal point of view and facilitate expression of beliefs and attitudes left undeveloped in an individual deep interview.

\section{Context}

Three PHCCs were purposefully sampled from a wide range of national PHCCs using the platform. Samples were chosen to provide a mix of urban and rural settings, as well as smaller and larger panel sizes. In each sampled PHCC, all GPs and nurses with experience of using the platform were invited to participate, with the goal of recruiting a minimum of six participants per group with an even distribution of GPs and nurses.

Participants gave written consent to participate in the focus group interview.

\section{Patient and public involvement}

Patients or the public were not involved in this study.

\section{Data availability statement}

Interview transcripts and coding data is available on request.

\section{Data collection}

Interviews were conducted between $5^{\text {th }}$ and $12^{\text {th }}$ June 2019 with a moderator (VMN) introducing topics with openended interview-guide questions (online supplementary appendix 1), facilitating the discussion with follow-up questions and summaries to verify interpretations. The interview guide was iteratively modified in response to evolving study findings. For data triangulation, an interview assistant (AE) observed and registered non-verbal communication but also aided the moderator in facilitating the discussion. Demographic data and quantitative data on months of experience working with the platform were also collected from all interview participants with a short questionnaire. Interviews were audio recorded (Olympus VN-8700PC) and transcribed verbatim.

\section{Data analysis}

Qualitative content analysis as presented by Graneheim and Lundman ${ }^{16}$ was used as it is a suitable inductive approach for describing human experience while also allowing for triangulation of analysis by researchers without contact with studied persons. ${ }^{17}$ Analysis was conducted in Swedish with NVivo 12. Relevant quotes were translated into English. The first author (AE) coded the data set (examples given in table 1), with regular discussions with two other authors (VMN and BBB) at all levels of analysis. All three authors where involved in thematisation. The manuscript was drafted using the Standards for Reporting Qualitative Research guidelines. ${ }^{18}$

Table 1 Examples of meaning units, condensed meaning units and codes

\begin{tabular}{lll}
\hline Meaning unit & Condensed meaning unit & Code \\
\hline $\begin{array}{l}\text {...if it has any medical consequences, it's too soon } \\
\text { to tell, there's too few, a too small sample }\end{array}$ & $\begin{array}{l}\text { Too small sample to know medical } \\
\text { consequences }\end{array}$ & Medical consequences unknown \\
$\begin{array}{l}\text {... and to be able to consult colleagues and the } \\
\text { doctors and such... I see that as positive, compared } \\
\text { with using the phone }\end{array}$ & $\begin{array}{l}\text { Easier to consult colleagues } \\
\text { compared with the phone }\end{array}$ & Enables colleague consultation \\
\hline
\end{tabular}


Table 2 PHCC, staff and interview characteristics

\begin{tabular}{|c|c|c|c|c|c|c|c|c|}
\hline & $\begin{array}{l}\text { Interview } \\
\text { duration } \\
\text { (min) }\end{array}$ & Location & $\begin{array}{l}\text { Patients } \\
\text { managed }\end{array}$ & $\begin{array}{l}\text { Number of staff } \\
\text { (as cited) }\end{array}$ & $\begin{array}{l}\text { Age } \\
\text { group }\end{array}$ & $\begin{array}{l}\text { Number of } \\
\text { females }\end{array}$ & $\begin{array}{l}\text { Mean years } \\
\text { with license } \\
\text { (range) }\end{array}$ & $\begin{array}{l}\text { Mean months } \\
\text { in platform } \\
\text { (range) }\end{array}$ \\
\hline \multirow[t]{2}{*}{ PHCC 1} & 49 & Urban & 9000 & $\begin{array}{l}3 \text { Nurses (Nurse } \\
1-3)\end{array}$ & $20-50$ & 3 & $4.3(3-5)$ & $2.7(2-3)$ \\
\hline & & & & $\begin{array}{l}1 \mathrm{GP} \\
(\mathrm{GP} 1)\end{array}$ & $50-60$ & 1 & $18(18-18)$ & $4(4-4)$ \\
\hline PHCC 2 & & & & $\begin{array}{l}3 \text { GPs } \\
(\text { GP 2-4) }\end{array}$ & $40-50$ & 1 & $10(9-11)$ & $4(1-6)$ \\
\hline PHCC 3 & 39 & Rural & 8000 & $\begin{array}{l}5 \text { Nurses (Nurse } \\
6-10)\end{array}$ & $30-60$ & 4 & $17.4(1-31)$ & $3.5(2-4)$ \\
\hline
\end{tabular}

GP, general practitioner; PHCC, primary healthcare centre.

\section{RESULTS}

\section{Study unit characteristics}

Characteristics of PHCC participants and the interviews are summarised in table 2 .

During analysis, 14 subcategories emerged, grouped into five categories, abstracted into two themes: 'Adjusting to a novel medium of communication' and 'Digitally filtered primary care' (table 3). Below, each category is described in detail.

\section{Fears and Benefits of Digital Communication}

Participants expressed an ambivalence towards the use of digital communication. Some felt curious and excited, while others expressed scepticism to the usefulness of such technology. PHCC 1 and PHCG 3 had relatively few patients using the platform, while PHCC 2 used the platform extensively. All participants felt it was too early to evaluate long-term risks and consequences of its use.

Nurses from the two urban PHCCs felt that the platform allowed patients to fully express their concerns without interruption, as some text presented by the automated patient interview was directly written by the patient.

And it's really their words. It's not our interpretation of their words. That's also... it becomes more certain, I think. - Nurse 3

Staff perceived an advantage of using software to ensure that relevant questions were always asked, without individual stress or other externalities affecting the

Table 3 Themes, categories and subcategories

\begin{tabular}{lll}
\hline Theme & Category & Subcategory \\
\hline $\begin{array}{l}\text { Adjusting to a novel medium of } \\
\text { communication }\end{array}$ & Altered Practice Workflow & $\begin{array}{l}\text { Streamlined communication } \\
\text { Improved interdisciplinary cooperation } \\
\text { Unpredictable workload }\end{array}$ \\
& Accepting the Digital Society & Expectations to be digital \\
& & Improved digital experience over time \\
& Safe and Secure for Patients & Improved management of certain patient \\
& & groups \\
Digitally filtered primary care & Accessible continuity \\
& Doesn't Suit Everyone and Everything & Not suitable for all patient queries \\
& & Digital communication as a partial \\
& solution \\
& Fears and Benefits of Digital & An incomplete system \\
& Communication & Incomplete information transfer \\
& & Ambivalence and uncertainty \\
& Superhuman capacity
\end{tabular}


consultation. The ability to reflect over messages before sending them was perceived as beneficial, especially for emotionally loaded discussions. On the contrary, staff highlighted that some patients experienced the chat as 'robotic', speculating that this could affect the patientprovider relationship.

Several participants mentioned that the automated patient interview allowed for acquisition of patient history data beyond what would otherwise be feasible during a regular phone call. While the presented information was perceived as useful, covering important differential diagnoses, staff felt overwhelmed for clinical decisionmaking. There seemed to be a reluctance towards overinformation, with GPs from PHCC 2 concluding that the most valuable information came from the first three free-text questions about patient ideas, concerns and expectations.

...it's about having just enough information in those questionnaires so that one can digest it... there is a balance... between too much and too little information too, so that it stays relevant... - GP 2

The platform was perceived to provide a unique value through the asynchronous chat, as clinical decisions could be communicated with several short messages without excessive conversation. Sending images was perceived to be useful, providing a unique benefit over telephone consultations, especially for dermatological queries. The platform did not include synchronous video consultations at the time, but these were speculatively perceived as less beneficial, as they were thought to too similar to telephone consultations.

One aspect is the automated patient interview tool and the other is the asynchronous communication. So those two things are new... I almost think that the asynchronous communication is the biggest benefit. I do. - GP 4

All groups felt that communicating via text led to some loss of communication nuance. One GP repeatedly emphasised the shortcomings of written communication, giving the impression of being particularly cautious about widespread use of this new technology. While facial expressions and body language were already absent in telephone consultations, cues like tonality were further removed when moving to text-based communication. Staff felt that these cues, in certain situations, provided important 'between the lines' context for interpretation of the reported symptoms.

That's probably why... fully artificial-intelligence-run systems refer 15 per cent to the emergency department... Because if one interprets peoples' words literally, then the whole healthcare system crashes. - GP 4

Patient interpretations of symptoms were perceived to not always be in-line with clinician interpretations. Misunderstood questions were not reformulated by the automated patient interview as would otherwise be possible in a live conversation.

What does 'dizziness' mean? ... There are many terms that mess things up. Because we're talking about different things, a certain symptom is one thing for the patient and another for me... so it's hard to just ask specific questions in a questionnaire like that. - GP 2

Most often, staff experienced symptoms to be less severe than reported when asking follow-up questions. GPs feared trivialising patient symptoms over time. Such risks were perceived lower with telephone consultations where severity was more confidently assessed. Consequently, some GPs felt that they tended to ask more follow-up questions via the platform compared with telephone consultations.

Yes, because I'm thinking if you look at the group presenting with anxiety and depression, for example, they get a lot of questions and then many of them specifically report suicidality or such, and... when one calls them, it isn't at all like they have written. - Nurse 4

The human ability to scrutinise reported information when consulting patients was deemed as central to the consultation process, but the automated patient interview was perceived to lack this ability.

In a conversation... one consciously ignores some things... Here it's 'on print'... that they have 'numbness in half of their body'... which looks a little worse than if they say it in a context where it is completely obvious that they don't... The 'human filter', it vanishes. - GP 4

Staff also expressed frustrations over being involuntarily responsible for irrelevant symptoms reported by the platform, including obsolete chronic symptoms or symptoms indicative of potentially severe disease.

Do you have abdominal pain?' Yes... they have had abdominal pain for 50 years. But we don't need to talk about that today. I would never ask the question in a normal conversation... or an obvious tension headache, but... visual impairment, asymmetrical pupil size... like "Aha, maybe we should order an ambulance instead?! - GP 3

This resulted in divergent agendas between GPs and patients where GPs focussed on addressing irrelevant but potentially urgent symptoms, while patients expected to get their primary less urgent concern addressed.

...it's not the questions I want the answer to, but which I have to assess... and it's extremely annoying... and now there's also a pop-up... saying that I am responsible for all the information I'm getting... Then I feel [the platform] limits me... that it takes longer than if I had done it another way. - GP 3 
Frustrations were also expressed regarding patients skipping questions, not reading staff responses, taking hours to answer follow-up questions or failing to confirm suggested appointments.

\section{Altered Practice Workflow}

In all PHCCs, nurses initially managed most queries in the platform. Staff from PHCC 2 estimated that around 30\% of queries were forwarded to GPs for further evaluation. Initially several nurses experienced stress of using the platform in addition to keeping track of electronic health records and other digital systems, as well as managing multiple parallel queries, especially when combined with physical visits and telephone consultations.

First it was a bit easy to make mistakes...if one had maybe five ongoing queries and maybe two girls around the same age or so to speak, it was easy to write to the wrong patient. ... until one develops a routine. - Nurse 1

However, staff generally felt that they handled digital queries faster and better over time. Miscommunication prevention, adjusting staffing at other workstations, scheduling adjustments and stress management strategies were examples of ongoing adjustments. The platform was then perceived as adding variation to the workday. There was a general sense that staff were content with the current state of affairs after a relatively hectic initial implementation of the new technology. Some PHCCs assigned rooms for work with the platform, with staff appreciating a less noisy environment.

All groups experienced shorter and more streamlined consultations, with easier appointment booking, information sharing and expressed reluctance of no longer having to redial patients not answering their phones.

...visits are better prepared and that's both good and bad. For example... someone seeking care for mental illness, who has already filled in rating scales etc, one enters the conversation at a different point. It's not like, 'Good day, what are you here for?'. Instead you have a lot of information before, when one starts the conversation... if it has any medical consequences is too soon to tell... - GP 9

Many felt that the chat-format made it easier to consult colleagues and gather information before answering certain patient queries, improving the interdisciplinary collaboration and the perceived working environment.

Challenges still remained, as staff expressed that certain patients took several hours to respond. By the end of the day, potentially urgent symptoms may thus have been left unaddressed. PHCC 3 managed this with a standardised message, informing patients to seek out-of-hours clinics for urgent symptoms.

\section{Accepting the Digital Society}

There was a general perception that digitalisation was not a choice. Parallels were drawn to implementation of telephone communication in family medicine, and pressures to use existing means of communication.

... if you have an entire panel who speaks English, then it's reasonable that we also speak English... we can't close our eyes to the fact that people communicate this way. We can't say 'we don't use phones, we use messages in bottles'... We have to adapt... - GP 4

Patients using the platform were perceived as being different from those seeking traditional care, with patients expecting fast responses, similar to a commercial customer support chat. Despite the challenges of adapting to the digital era, there was a general sense that the platform was perceived better over time.

When it came we were a bit scared that it would be a lot... that we wouldn't be able to handle it, but today I feel that we are all pretty positive and that we more easily can communicate with patients and it will only get easier - Nurse 7

In fact, all practices expressed a desire to stay digital, with two PHCCs incentivising patients to use the platform by offering shorter waiting time for appointments or automatically redirecting certain patients from the phone.

\section{Safe and Secure for the Patient}

The platform was perceived to aid in triage by giving an overview of incoming presenting symptoms and reported symptoms. There was a general perception of improved access to care as staff felt that patients more quickly could engage in dialogue with nurses compared with telephone visits.

Many appreciate that $100 \%$ availability which it really provides. [Patients] can write and will get through... that's very reassuring - GP 2

Staff were also surprised that the platform was occasionally used by elderly individuals and patients with socioeconomic difficulties.

It was a patient who otherwise has a very strained life. I was very surprised that she could use it, but it's worked well for her... a single mother with three small children... working full-time and finds phone calls from the practice difficult during working hours... So we can send her a text, or chat with her and manage things when it works for her... She thought it was great. - GP 1

PHCC 2 experienced a transition from initially viewing the platform as a triage tool to a tool for improving continuity of care, giving the PHCC a unique advantage over private 'digital only' family medicine providers. One GP felt that his frequent visitors could be managed more effectively with chat follow-ups. Following stable chronic conditions, selective serotonin reuptake inhibitor treatments and dermatological diagnoses were other examples of platform use for improved continuity. Staff were 
uncertain, however, whether the platform had substantially reduced physical visits in general.

...I perceive that for my patients, mostly the sickest or most worried ones, it's a huge reassurance and very personal. When they can chat with me and I can say like 'We don't need to book a new appointment'... 'Take it easy and be in touch. It may take a day before I answer, but I will answer.'... then they have a face associated to the person writing... then one can sometimes even crack a joke in the chat - GP 4

\section{Doesn't Suit Everyone and Everything}

All groups acknowledged that digital communication didn't suit all patient queries. Although some technically literate elderly patients used the platform, staff felt others were less confident often resulting in phone calls being made to clarify the issue. Staff generally felt the patients with simple queries were manageable in the platform, while complex queries or cases of low continuity were situations where the platform was perceived as less useful. In multiple instances, staff explained that queries which required prolonged dialogue via text often resulted in a phone call as this was perceived as a more effective way of managing and concluding such queries.

A number of technical improvements were lifted to adapt the platform to local prerequisites.

Many queries are pretty simple... 'I want to renew a prescription', 'what did my tests show?', 'why is there such a long waiting time'. In these situations, one isn't dependent on any finessed nuances... - GP 9

GPs envisioned digital communication as an additional tool to existing ways of working. Few queries were managed completely digitally, but rather 'digi-physically' as digital communication could on many occasions contribute to overall management of a patient, followed by an occasional physical examination. Classification into digital or physical care was thus seen as a false dichotomy, as transitioning between modes of communication often was perceived as useful depending on the clinical situation.

\section{DISCUSSION}

\section{Main findings}

PHCC staff initially experienced implementation of the platform as both uncertain and exciting. Over time, views of the platform seemed to shift from a foreign entity with a specific purpose to an integrated part of practice complementing other modes of patient communication. Challenges remained, but there was a general sense that staff wished to remain digital.

\section{Themes}

The theme 'adjusting to a novel medium of communication' highlights how staff experienced having to accept and integrate asynchronous communication into practice, but also experiencing value in management of certain patients as well as improved continuity.

The theme 'digitally filtered primary care' highlights that staff experienced patient data presented both in overwhelming detail in terms of symptom reports, but also with loss of communication nuances which created an uncertainty in the management of some patients.

\section{General discussion}

Our findings conceptualise digital communication as both an alternate means of information exchange (a transactional process) as well as a means of developing and maintaining doctor-patient relationships (a transformational process), two dominating paradigms in the communication literature ${ }^{19}$ Additionally, implementing digital communication had effects beyond patient communication, that is, on practice organisation and working environment.

Qualitative research on primary care staff experiences of implementing automated patient interview software combined with two-way asynchronous digital communication is limited. Johansson and Ivarsson recently presented survey data on nurse experiences of a pilot version of the platform. ${ }^{20}$ Like our study, they found that nurses experienced improved triage, high patient satisfaction, issues of care supply to specific patient populations and issues with managing information technology systems. ${ }^{20}$ Our results add depth to these findings, as well as focussing primarily on staff experiences of digital communication beyond the platform itself.

In a separate publication, Johansson et al interviewed GPs after two months of using the same pilot platform. ${ }^{21}$ Similar to our study, GPs expressed that the patients' selfreported medical history and asynchronous communication had a unique benefit, that visits were well prepared and that collegial collaboration increased. Furthermore, the GPs experienced that symptom severity was difficult to assess, that working with multiple IT systems was cumbersome and that not all queries were suitable. Our study adds staff experiences past two months of using the fully developed version of the platform, where staff express wishing to stay digital and further integrate the platform into practice.

Unlike our study, other studies have found that GPs experienced digital communication as poorly integrated into clinical practice, adding to increasing workloads. ${ }^{89}$ These were platforms without two-way communication and patient-centred questionnaires, and queries weren't triaged by nurses prior to reaching GPs, indicating that our findings are context-specific.

Our findings are consistent with a Cochrane review concluding that health workers felt that two-way textbased communication can facilitate the patient-provider relationship, but that specific situations still warrant faceto-face consultations. ${ }^{13}$

The finding that two-way digital communication focusses queries while letting patients better express 
their concerns is consistent with studies on nurses in the context of prostate cancer management. ${ }^{10}$

The risk of misunderstandings given two-way written digital communication has also been expressed by clinicians in the context of managing diabetes ${ }^{11}$ and young people with long-term conditions. ${ }^{12}$ The last study also concluded that digital communication is best implemented when there is an existing patient-provider relationship of trust. ${ }^{12}$ Continuity of care thus remains a central component of a highly functioning primary care system. ${ }^{22}$

\section{Strengths}

Several factors add to the trustworthiness of our findings. First, credibility increased by prolonged engagement, peer debriefing from coding to categorisation and data triangulation with non-verbal observations. The two interviewers had experience with using digital communication in primary care, creating a mutual understanding of the context the participants worked with. Investigator triangulation with a third researcher without a background in digital communication added an alternative perspective on the data for a richer interpretation. Highly engaged participants allowed for thorough descriptions of our goal phenomenon, adding transferability of our findings to similar contexts. Purposefully sampled PHCCs from both rural and urban settings added generalisability to our findings.

No new subcategories emerged from the final focus group, suggesting that 'theoretical saturation' was reached ${ }^{23}$ However, we cannot exclude that further focus groups would yield a different final perspective.

\section{Limitations}

Due to limited resources, we were unable to conduct secondary coding. We didn't conduct member checks which limits credibility. Lack of an audit trail also limits confirmability and consistency. This was a small study with three PHCCs and thus the experiences described may not represent those of most staff using the platform. The technology is new, and presumably currently adopted by PHCCs interested in using it. ${ }^{24}$

Mixing GPs and nurses may have influenced the results as GPs in some focus groups were perceived to answer more readily than nurses. However, mixing groups also allowed for instant exploration of experiences shared by both professions. Finally, as interviewers were both GPs, participant engagement and interpretation of results may have been skewed in favour of GP over nurse perspectives.

\section{CONCLUSIONS}

Family medicine staff experience a period of adjustment to integration of digital communication in a time when such communication is extensively used and expected by patients. Despite concerns about inappropriate use and difficulties interpreting text, staff experience digital communication as a potentially useful choice of communication in certain contexts, especially when combined with continuity of care. Future research should explore which specific clinical contexts are best suited for digital communication.

\section{Twitter Artin Entezarjou @doctorartin}

Acknowledgements We would like to thank the practice managers, general practitioners and nurses for sharing their valuable experiences.

Contributors AE, VMN, SC and PM conceptualised the study. AE and VMN conducted data collection. AE, VMN and BBB contributed to analysis of the results. All authors contributed to the preparation of the manuscript.

Funding This study was funded by the Thelma Zoéga's fund and Region Skåne to Artin Entezarjou and funding from ALF and Region Skåne to Susanna Calling. Funders had no role in data collection, interpretation or reporting.

Competing interests None declared.

Patient and public involvement Patients and/or the public were not involved in the design, or conduct, or reporting, or dissemination plans of this research.

Patient consent for publication Not required.

Ethics approval The study was approved by the Swedish Ethical Review Authority (reference number 2019-01516).

Provenance and peer review Not commissioned; externally peer reviewed.

Data availability statement Data are available upon reasonable request. Interview transcripts and coding data is available upon request.

Open access This is an open access article distributed in accordance with the Creative Commons Attribution Non Commercial (CC BY-NC 4.0) license, which permits others to distribute, remix, adapt, build upon this work non-commercially, and license their derivative works on different terms, provided the original work is properly cited, appropriate credit is given, any changes made indicated, and the use is non-commercial. See: http://creativecommons.org/licenses/by-nc/4.0/.

\section{ORCID iDs}

Artin Entezarjou http://orcid.org/0000-0002-7418-8750

Beata Borgström Bolmsjö http://orcid.org/0000-0001-7655-0613

Susanna Calling http://orcid.org/0000-0002-2420-3780

Patrik Midlöv http://orcid.org/0000-0002-5871-8731

Veronica Milos Nymberg http://orcid.org/0000-0002-3836-3048

\section{REFERENCES}

1 Ekman B, Thulesius $\mathrm{H}$, Wilkens J, et al. Utilization of digital primary care in Sweden: descriptive analysis of claims data on demographics, socioeconomics, and diagnoses. Int J Med Inform 2019;127:134-40.

2 Organisation for Economic Co-operation and Development (OECD). Health at a glance 2019: OECD indicators. Paris: OECD publishing, 2019.

3 Vårdanalys. Vården ur primärvårdsläkarnas perspektiv - en jämförelse mellan Sverige och nio andra länder [Healthcare from general practicitioner perspectives - a comparison between Sweden and nine other countries, 2015.

4 Atherton H. Digitally enabled primary care: the emperor's new clothes? Br J Gen Pract 2019;69:420-1.

5 Scott Kruse C, Karem P, Shifflett K, et al. Evaluating barriers to adopting telemedicine worldwide: a systematic review. J Telemed Telecare 2018;24:4-12.

6 Larsen J-H, Neighbour R. Five cards: a simple guide to beginning the consultation. Br J Gen Pract 2014;64:150-1.

7 Fagerlund AJ, Holm IM, Zanaboni P. General practitioners' perceptions towards the use of digital health services for citizens in primary care: a qualitative interview study. BMJ Open 2019;9:e028251.

8 Banks J, Farr M, Salisbury C, et al. Use of an electronic consultation system in primary care: a qualitative interview study. $\mathrm{Br} J$ Gen Pract 2018;68:e1-8.

9 Cowie J, Calveley E, Bowers G, et al. Evaluation of a digital consultation and self-care advice tool in primary care: a MultiMethods study. Int J Environ Res Public Health 2018;15:15050896.

10 Clarke AL, Roscoe J, Appleton R, et al. My gut feeling is we could do more... a qualitative study exploring staff and patient perspectives 
before and after the implementation of an online prostate cancerspecific holistic needs assessment. BMC Health Serv Res 2019;19.

11 Lie SS, Karlsen B, Graue M, et al. The influence of an eHealth intervention for adults with type 2 diabetes on the patient-nurse relationship: a qualitative study. Scand J Caring Sci 2019;33:741-9.

12 Griffiths F, Bryce C, Cave J, et al. Timely digital Patient-Clinician communication in specialist clinical services for young people: a mixed-methods study (the LYNC study). J Med Internet Res 2017;19:e102.

13 Odendaal WA, Anstey Watkins J, Leon N, et al. Health workers perceptions and experiences of using mHealth technologies to deliver primary healthcare services: a qualitative evidence synthesis. Cochrane Database Syst Rev 2020;3:Cd011942.

14 Creswell JW, Poth CN. Qualitative inquiry and research design choosing among five approaches. 4th ed. Thousand Oaks: SAGE Publications Inc, 2018.

15 Kitzinger J. Qualitative research. introducing focus groups. BMJ 1995;311:299-302.

16 Graneheim UH, Lundman B. Qualitative content analysis in nursing research: concepts, procedures and measures to achieve trustworthiness. Nurse Educ Today 2004;24:105-12.

17 Graneheim UH, Lindgren B-M, Lundman B. Methodological challenges in qualitative content analysis: a discussion paper. Nurse Educ Today 2017;56:29-34.
18 O'Brien BC, Harris IB, Beckman TJ, et al. Standards for reporting qualitative research: a synthesis of recommendations. Acad Med 2014;89:1245-51.

19 Manojlovich M, Squires JE, Davies B, et al. Hiding in plain sight: communication theory in implementation science. Implement Sci $2015 ; 10: 58$

20 Johansson A, Ivarsson B. Nurse Telephone Counseling Services as a "Gatekeeper" in an Internet-Based Digital Doctor Reception: A Mixed Questionnaire Survey. J Prim Care Community Health 2019;10:2150132719886952

21 Johansson A, Larsson M, Ivarsson B. General practitioners' experiences of digital written patient Dialogues: a pilot study using a mixed method. J Prim Care Community Health 2020;11:215013272090965.

22 Pereira Gray DJ, Sidaway-Lee K, White E, et al. Continuity of care with Doctors-a matter of life and death? A systematic review of continuity of care and mortality. BMJ Open 2018;8:e021161.

23 Saunders B, Sim J, Kingstone T, et al. Saturation in qualitative research: exploring its conceptualization and operationalization. Qual Quant 2018;52:1893-907.

24 Rogers EM. Lessons for guidelines from the diffusion of innovations. Jt Comm J Qual Improv 1995;21:324-8. 